\title{
Multisorted Tree-Algebras for Hierarchical Resources Allocation
}

\author{
Erick Patrick Zobo and Marcel Fouda Ndjodo \\ Department of Computer Science and Educational Technology, Higher Teachers Training College, \\ University of Yaoundé I, Yaoundé, Cameroon \\ Correspondence should be addressed to Erick Patrick Zobo; epzobo@dite-ens.cm
}

Received 2 December 2014; Revised 13 April 2015; Accepted 29 April 2015

Academic Editor: Robert J. Smith?

Copyright ( 2015 E. P. Zobo and M. Fouda Ndjodo. This is an open access article distributed under the Creative Commons Attribution License, which permits unrestricted use, distribution, and reproduction in any medium, provided the original work is properly cited.

\begin{abstract}
This paper presents a generic abstract model for the study of disparities between goals and results in hierarchical multiresources allocation systems. In an organization, disparities in resource allocation may occur, when, after comparison of a resource allocation decision with an allocation reference goal or property, some agents have surplus resources to accomplish their tasks, while at the same time other agents have deficits of expected resources. In the real world, these situations are frequently encountered in organizations facing scarcity of resources and/or inefficient management. These disparities can be corrected using allocation decisions, by measuring and reducing gradually such disparities and their related costs, without totally canceling the existing resource distribution. While a lot of research has been carried out in the area of resource allocation, this specific class of problems has not yet been formally studied. The paper exposes the results of an exploratory research study of this class of problems. It identifies the commonalities of the family of hierarchical multiresource allocation systems and proposes the concept of multisorted treealgebra for the modeling of these problems. The research presented here is not yet an in-depth descriptive research study of the mathematical theory of multisorted tree-algebra, but a formal study on modelling hierarchical multiresource allocation problems.
\end{abstract}

\section{Introduction}

Hierarchical multiresource allocation (HMRA) is the process of assigning a multiresource to operating agents through a decision making hierarchy made up of management agents [1-5]. Each management agent is supposed to allocate optimally the available resources to its immediate subordinate management or operating agents $[6,7]$ by taking into account their specific needs and the policy of the organization. Examples of this kind of resource allocation processes abound in the literature for allocation of personnel [8], allocation of facilities [9], allocation of items or services [10], and allocation of suppliers or equipment [11].

The multiresource allocation problems presented in the literature $[12,13]$ usually concern the optimization of social welfare of operating agents or the measurement of fairness and/or efficiency of the allocation decisions. However, in many real world situations as in organizations, resource allocation decisions are usually taken in consideration of the resource previously allocated to agent. This is quite different from the operation research literature $[8,12,14]$, where an agent requesting the resources does not own any resource before the allocation decision of the allocator. Here resource allocation decisions are taken to improve the existing allocation state efficiency after measuring their disparities and consequences, with the objective of reducing them gradually by new allocation decisions. These disparities measurement may correspond to the utility functions of the agents but instead be a quest of the management board of the organization.

The main concern of this work is to build a mathematical framework appropriate for modelling and studying performance measurement problems of multiresource allocation decisions in hierarchical organizations where each agent has a capacity to consume a given quantity of resource. That quantity generally depends on its role for achieving the organizations service or goal. In this situation, the level of performance of an allocation decision is reflected by the disparity of that allocation. This disparity is obtained by comparing the current state of resource allocation capacity with 
a resource allocation reference state or property. The obvious importance of this concern in organizations is that resource disparities generate overcosts and therefore have a negative impact on the expected quality of service. One can notice that computation of these disparities in a hierarchical system is similar to information fusion approaches as presented in $[15,16]$.

The paper introduces a formal conceptual framework, referred to as multisorted tree-algebra, and proposes an abstract generic model for the study of disparities and their related problems in HMRA systems. It identifies and defines five basic commonalities of this class of problems: (i) allocation environment (service, multiresources, agents, and hierarchy), (ii) allocation policy (resource utilization, agent request, and allocation quality goals), (iii) decision framework (allocation, reallocation, and deallocation), (iv) analysis framework (perspective, reference, and disparities), and (v) generic problems (decision problems, optimization problems).

The paper is organised as follows. Section 1 introduces the HMRA model; Section 2 highlights the basic components of HMRA systems; Section 3 introduces the modeling formalism; Section 4 presents HMRA system model; Section 5 presents a case study on teachers allocation disparities in Cameroon's secondary education system discusses the model; and Section 6 concludes the paper.

\section{Hierarchical Multiresources Allocation (HMRA) Systems' Basic Components}

In this section we describe the four components of a generic policy-based hierarchical multiresource allocation framework. These components include the environment described in Section 2.1; the policy given in Section 2.2; the analysis criteria in Section 2.3; and the generic problems in Section 2.4. The framework has similarities with the hierarchical multiresource allocation described by Van Zandt $[2,17]$, but it does not study real-time communication costs.

2.1. HMRA Environment. An hierarchical multiresource allocation environment is the organisation structural components used to define its multiresource allocation decisions and procedures. An HMRA environment is composed of the following five components: the organisations service; the resources; the hierarchical shape; the operating agent; and the management agent. These components are described with their related notions as follows.

(i) The Service. An organisation service is the set of all the atomic tasks its operating agents are supposed to achieve during a period of time. A subset of an organisation's service is called an activity. Hence two different activities can contain one or many identical tasks. A set of activities represents a duty or program that can be assigned to an organisation's operating agent.

(ii) The Resources. Organisations' resources are entities used by operating agents to perform organisations' tasks $[8,10,18]$. The resource features are the set of atomic modalities used to describe a resource. A subset of resource features is called a resource character. A set of resource characters is called a resource portrait. A resource type is a resource character for which each modality corresponds with an organisations task to indicate in which task the resource can be allocated.

(iii) The Hierarchy Shape. An organisation's hierarchy shape is a tree where nodes represent the organisation's components as shown in Figure 1. The root represents the general manager, the intermediate nodes represent management agents/units, and the leaves represent operational agents/units. The edges of that shape represent the hierarchical relationships between the organisation's agents. Given a tree, the length of a leaf is the number of edges from the root to that leaf. For example, the length of leaves of the tree in Figure 1 is 3. A tree is balanced when all its leaves have the same length. The hierarchy shapes considered in this paper are assumed to be balanced. Such hierarchical shapes are most often observed in governmental organisations like education systems, military systems, and health systems $[19,20]$.

(iv) The Operating Agent. An operating agent is an organisation unit placed at the bottom of the organization's hierarchy that requests a quantity of resources necessary to achieve its assigned duty. An operating agent is characterized by three components: its profile, its capacity, and its potential.

(a) An operating agent profile is a couple with components corresponding to the agent's assigned duty and the agent's size. An agent's duty is the set of its assigned activities or tasks to be achieved within a period of time. An agent's size is a vector of integers that represents the number of iterations of each of its assigned activities.

(b) An operating agent capacity is the agent's total workload hours of each task that it could achieve within a given period of time, when all the requested resources are supplied.

(c) An operating agent potential is the agent's effective workload it could achieve within a given period of time with the currently supplied resources.

(v) The Management Agent. A management agent is an organization unit placed at the intermediary nodes of the organization's hierarchy, which is in charge of resource allocation decisions. They allocate resources to their subordinate resource management units or operating agents. A management agent can also be considered as an operating agent for which allocated resources are the aggregation of resources that are allocated to all its subordinate operating agents. A management agent is therefore characterized by the similar components characteristic of an operating agent, which are its profile, its capacity, and its potential. They are defined as follows:

(a) A management agent profile is the aggregation of all its subordinate operating agent profiles.

(b) A management agent capacity is the aggregation of all the subordinate operating agent capacities. 


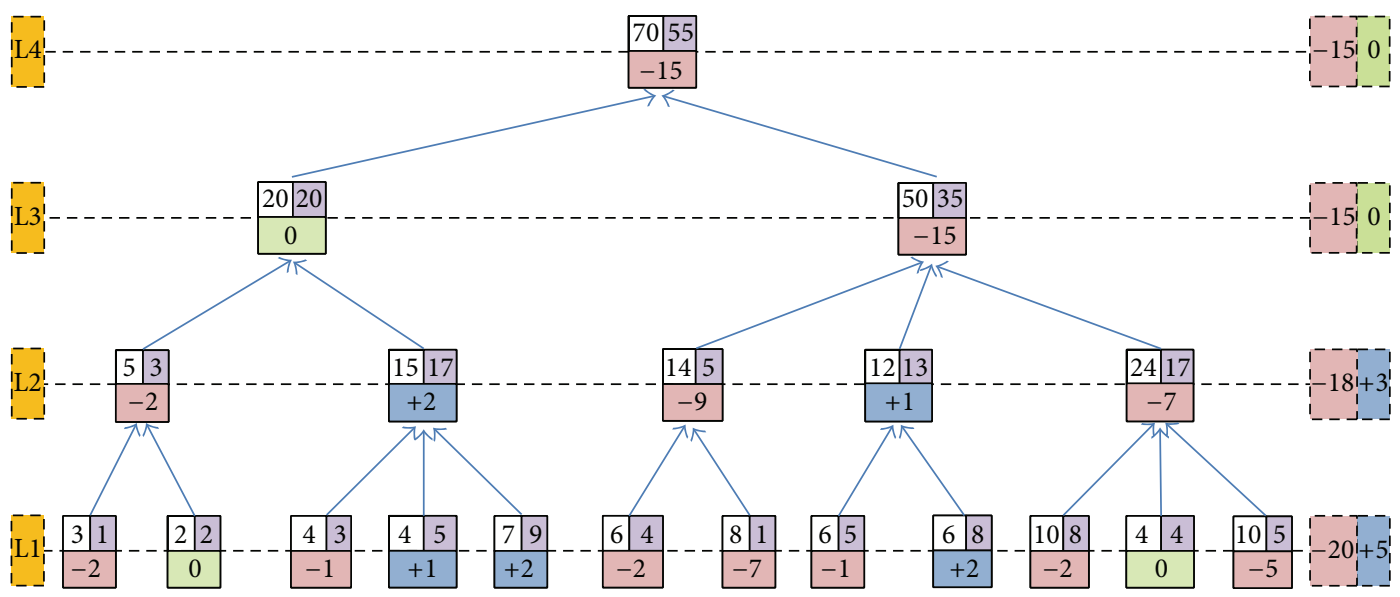

FIGURE 1: Example of hierarchical structure with resources allocation decision levels in colored boxes.

(c) A management agent potential is the aggregation of all the subordinate operating agent potentials.

2.2. The HMRA Policy. In this section we describe the two main generic components of an hierarchical multiresource allocation policy. They are the resource policy components and the allocation policy components.

(i) Multiresource Policy Components. A multiresource policy component is a set of parameters used to describe a multiresource allocation decision. The two main generic multiresource policy components are defined as follows:

(a) A multiresource management portrait is a set of resource features used as parameters for constructing resource allocation decisions and analyses. It is a subset of an organisation's resource portraits that defines a perspective from which resources are managed.

(b) A multiresource utilisation convention is a set of relations that defines how the resources are supposed to be used by the operating agents. They specify, for instance, their workload within a period, their functioning costs within the same or a different period, and their utilization longevity within the organisation. These conventions take into account the resource types and their features together with the activities or task loads.

(ii) Allocation Policy Components. An allocation policy component is a set of parameters used to define a resource allocation decision. In this paper, the following are generic allocation policy components identified:

(a) an allocation perspective is a set in which elements represent the various features from which resource allocation decisions are defined;

(b) an allocation procedure is the set of steps to follow when allocating resources; (c) an allocation decision is the end result of allocation procedures that describe for each operating agent its allocated resources.

(iii) Configuration. A resource allocation configuration is a function that assigns to each organisation's agent the set of configuration components used to analyse its resource allocation state.

(a) A configuration component is an indicator used to describe, analyse, or observe an agent's state after a resource distribution outcome. In a quantitative analysis perspective, this component may include an agent's capacity, potential, or performance.

(b) An operating agent configuration is the output of the configuration function for an operating agent.

(c) A management agent configuration is the output of the configuration function for a management agent corresponding to the aggregation of its subordinate operating agent configurations.

2.3. The Decision Framework. A resource allocation decision framework is the set of elementary decision types that could be taken or could be combined with management agents in the organization. These decision types are described as follows.

(i) Allocation. An allocation is a decision that adds more resources to the ones already distributed amongst the subordinate agents.

(ii) Reallocation. A reallocation is a decision that changes the distribution of resources amongst the subordinate operating agents without augmenting or reducing their number.

(iii) Disallocation. A disallocation is a decision that removes resources from the available resources distributed amongst the operating agents. 
2.4. The Analysis Criteria. A resource allocation analysis framework is the set of generic components used in analysing the resource distribution in an organisation. These components are described as follows.

(i) Analysis Perspective. An analysis perspective is a set of managerial criteria or features used to define analysis indicators. An example of perspective may be cost oriented, performance oriented, potential oriented, capacity oriented, or oriented towards any other isolated or combined managerial criteria.

(ii) Reference Property. A reference property is a description of the characteristics of a resource allocation result. These properties generally characterise a viewpoint of concepts broadly related to the notion of fairness or equity. For example, at a given hierarchical level of the organisation, an allocation decision satisfies the potential-based equality property when each agent has the same potential. The allocation decision satisfies the proportional-based potential property when there is a set of numbers such that the potential of agents associated with these numbers is proportional. An allocation decision satisfies the capacity-based proportional potential property when the agent potential associated with their capacity is proportional. An allocation decision satisfies the cost-based balance disparities property if the cost of disparities for each agent is zero.

(iii) Allocation Disparity. An allocation disparity of an operating or management agent is the difference between a reference property and its configuration components observed through a given perspective. In this paper, the allocation disparity of an agent is defined as the difference between its resources workload capacity (also called resources capacity) and the workload of its allocated resources (also called resources potential).

2.5. The Generic Problems. A generic problem is a class of resource allocation problems. The two main types of generic problems in the resource allocation framework are defined as follows:

(i) a decision problem is the class of resource allocation problems for which the challenge is to find within alternative resource allocation decisions, the one that satisfies a given set of constraints.

(ii) an optimization problem is the class of resource allocation problems for which the challenge is to get the maximum or minimum of the resource allocation decision alternatives that are solutions of the problem.

\section{The Modelling Formalism}

3.1. Portrait-Sets. In this section we introduce a modeling framework for features-based quantitative description of a finite set of resources. The motivation is based on the fact that a set of resources can be described from different viewpoints representing resource features or characters. These features are used as decision criteria in resource allocation decision formulation or analysis. In Section 3.1, the concept of portrait-sets is introduced as a modelling framework for features-based quantitative description of sets of resources. Here, a set of resources is associated with a set of characters representing various resource description viewpoints called portraits. Each character is a set of resources modalities that could be used as survey criteria on the set of resources. In Section 3.2, we introduce the concept of portrait-cardinality as the quantitative description of a given set (of resources) from a considered portrait. It gives a quantitative description of resources for each modality included in each character of the portrait. Depending on the number of characters in the portrait, a portrait-cardinality of a given set can be designed as a vector (for a portrait of one character), a matrix (for a portrait of two characters), or a volume (for a portrait of more than two characters).

Definition 1 (characters, modalities, and correlation character). Let $E$ be a nonempty set and $k$ a positive integer, $k \geq 2$. Let $X=\left\{x_{1}, \ldots, x_{n}\right\}$ be a set of $n$ elements.

(i) A $k$-character is a vector $C=\left(m_{1}, \ldots, m_{k}\right) \in \mathscr{M}(E$, $1, k)$ for which components from $E$ are called modalities. A character is quantitative when its modalities are reals or sets of reals and qualitative elsewhere. A character $C$ is sound when no element can satisfy two different modalities from $C$.

(ii) Let $C$ be a $k$-character. The set $X$ is a $C$-set or satisfies the character $C$ if there is a weak partition $X_{C}=$ $\left\{X_{i}\right\}_{i \in C}$ of $X$, where each component $X_{i}$ is the set of elements in $X$ that satisfies the modality $i$.

(iii) Let $A=\left(a_{1}, \ldots, a_{i}, \ldots, a_{k}\right) \in \mathscr{M}(E, 1, k)$ and $B=$ $\left(b_{1}, \ldots, b_{j}, \ldots b_{p}\right) \in \mathscr{M}\left(E^{\prime}, 1, p\right)$ be two characters. A 2-correlation character of $A$ and $B$ is the character denoted by $A \times B$ and defined by $A \times B=$ $\left(c_{11}, c_{12}, \ldots, c_{i j}, \ldots, c_{k p}\right) \in \mathscr{M}\left(E \times E^{\prime}, 1, k \times p\right)$, where the modality $c_{i j}=\left(a_{i}, b_{j}\right)$ represents the binary correlation of modalities $a_{i}$ and $b_{j}$. Each $c_{i j}$ is satisfied if $a_{i}$ and $b_{j}$ are simultaneously satisfied.

(iv) The set $X$ is said to be a $C$-partially free if there is at least one element of $X$ that does not satisfy any character in $C$.

(v) The set $X$ is said to be $C$-free if no modality from $C$ is satisfied by elements of $X$.

Definition 2 (portraits and portrait-sets). (i) A portrait is a set of characters. Let $l$ be a positive integer; an l-portrait is a set $P=\left\{C_{1}, \ldots, C_{l}\right\}$ of $l$ characters.

(ii) Let $X$ be a nonempty set. Let $P=\left\{C_{1}, \ldots, C_{l}\right\}$ be an $l$-portrait. The set $X$ is a $P$-portrait set or simply a $P$-set if $X$ is a $C_{i}$-set for each $i, 1 \leq i \leq l$.

Remark 3. If $X$ satisfies the portrait $\{A, B\}$, then it is also an $A \times B$-set and can be represented by either a vector $\left(v_{j}\right) \in$ $\mathscr{M}(\mathbb{N}, 1, k \times p)$ or a matrix $\left(y_{i j}\right) \in \mathscr{M}(\mathbb{N}, k, p)$ for which the component $v_{j}$ or $y_{i j}$ represents the cardinality of $X_{i j}$ from the partition $X_{A \times B}$. 
Generally, let $P=\left\{C_{1}, \ldots, C_{k}, \ldots C_{l}\right\}$ be a portrait and $X$ a $P$-set. The set $X$ has all the $k$-correlation characters from $P$ with $1 \leq k \leq l$ and can be represented by their corresponding vectors, matrix and for $1 \leq k \leq l$, by the multidimensional volumes $V_{X, k}^{P} \in \mathscr{M}\left(\mathbb{N}, n_{1}, \ldots, n_{j}, \ldots, n_{k}\right)$, where $n_{j}$ is the cardinality of $C_{j}$ with $1 \leq j \leq k$.

\subsubsection{Portrait-Cardinality}

Definition 4 (C-cardinality and $P$-cardinality). (i) Let $C$ be a $k$-character and $X$ a $C$-set. The set $X$ can be represented by a statistical series $\left\{\left(m_{1}, q_{1}\right), \ldots,\left(m_{t}, q_{t}\right), \ldots,\left(m_{k}, q_{k}\right)\right\}$ denoted by $\left|X_{C}\right|$, where for each $t, 1 \leq t \leq k$, the integer $q_{t}$ is the cardinal of $X_{t}$. The vector $\widetilde{X}_{C}=\left(q_{1}, \ldots, q_{t}, \ldots, q_{k}\right) \in \mathscr{M}(\mathbb{N}, 1, k)$ is called the Character $C$ cardinality or $C$-cardinality of $X$.

(ii) Let $P=\left\{C_{1}, \ldots, C_{l}\right\}$ be a portrait and let $X$ be a $P$-set. There is a weak partition $\left\{X_{k}\right\}$ of $X$ indexed with the elements $k \in \prod_{i=1}^{i=l} C_{i}$, where the set $X_{k}$ represents the set of elements of $X$ that satisfies simultaneously the modalities appearing in $k$. The portrait-cardinality or $P$-cardinality of the $P$-set $X$ is the family denoted by $\widetilde{X}_{P}$ of cardinals of $X_{k}$ for each $k \in \prod_{i=1}^{i=l} C_{i}$.

When the context is clear, $\widetilde{X}_{P}$ is simply denoted by $\widetilde{X}$. When $l=2$ and the cardinals of $C_{1}$ and $C_{2}$ are, respectively, $n$ and $u$, the $P$-cardinality of the $P$-set $X$ is represented by an $(n \times u)$-matrix $\widetilde{X} \in \mathscr{M}(\mathbb{N}, n, u)$. In this paper, we are interested in a portrait-set with at most two characters.

\subsubsection{Matrix Comparison, Fragmentation, and Aggregation}

Definition 5 (weak partition and partition). Let $E$ be a nonempty set. Let $\left\{A_{i}\right\}_{i \in I}$ be a family of subsets of $E$. The family $\left\{A_{i}\right\}_{i \in I}$ is a weak partition of $E$ if the following conditions are satisfied:

(i) For $i \in I$ and $j \in I$ such that $i \neq j, A_{i} \cap A_{j}=\emptyset$.

(ii) $\bigcup_{i \in I} A_{i}=E$.

When each component $A_{i}$ is a nonempty set, then the family $\left\{A_{i}\right\}_{i \in I}$ is a partition of $E$.

When the set $I$ is a finite set of $m$ elements, a weak partition $\left\{A_{i}\right\}_{i \in I}$ is identified as an element $\left(A_{1}, \ldots, A_{k}, \ldots, A_{m}\right)$ of $2^{E^{m}}$.

Definition 6 (comparison of matrices). Let $\left(E, \leq_{E}\right)$ be an ordered set. Let $A=\left(a_{i j}\right)$ and $B=\left(b_{i j}\right)$ be two matrices in $\mathscr{M}(E, n, p)$. There is an induced order $\leq_{\mathscr{M}(E, n, p)}$ defined in $\mathscr{M}(E, n, p)$ from the order $\leq_{E}$ as follows:

$A \leq \mathscr{M}(E, n, p) B$ if and only if $a_{i j} \leq b_{i j}$ for all $1 \leq i \leq n$ and $1 \leq j \leq p$. The matrix $A$ in this case is called a fragment of the matrix $B$.

When the context is clear, $\leq_{E}$ and $\leq_{\mathscr{M}(E, n, p)}$ are simply denoted by $\leq$.

The definition of matrix fragmentation below is given to capture the teacher distribution in a school system. Here, teachers in the school system are described by a matrix $M$, and they are distributed into schools where their corresponding description in each school is a fragment of $M$.

Definition 7 (matrix fragmentation). Let $(E,+, 0, \leq)$ be an ordered group structure. Let $m, n$, and $p$ be positive integers. Let $M_{0} \in \mathscr{M}(E, n, p)$ and let $F=\left\{M_{k}\right\}_{1 \leq k \leq m}$ be a family of matrices in $\mathscr{M}(E, n, p)$. The family $F$ is an $m$-fragmentation of $M_{0}$ if the following conditions are satisfied:

(i) $M_{k} \leq M_{0}$ for each $k$ such that $1 \leq k \leq m$;

(ii) $\sum_{k=1}^{k=m} M_{k}=M_{0}$.

The components of $F$ are called the fragments of $M_{0}$ in $F$ and $M_{0}$ is called the $m$-aggregation of $F$.

Definition 8 (matrix aggregation). Let $M_{0} \in \mathscr{M}(\mathbb{N}, n, p)$. The set of possible fragments of $M_{0}$ is denoted by $\mathscr{F}\left(M_{0}\right)$ and defined as $\mathscr{F}\left(M_{0}\right)=\left\{M \in \mathscr{M}(\mathbb{N}, n, p), M \leq M_{0}\right\}$. The set of all the $m$-fragmentation of $M_{0}$ is denoted by $\mathscr{F}_{m}\left(M_{0}\right)$ and defined by $\mathscr{F}_{m}\left(M_{0}\right)=\left\{\left(M_{i}\right)_{1 \leq i \leq m} \in\left(\mathscr{F}\left(M_{0}\right)\right)^{m}\right.$ with $\left.\sum_{k=1}^{k=m} M_{k}=M_{0}\right\}$.

Proposition 9 (inclusion and cardinality). Let $C$ be a portrait. Let $X, Y$ be two sets. If $X$ is a $C$-set and $Y \subseteq X$, then (a) $Y$ is a $C$-set and (b) $\widetilde{Y} \leq \widetilde{X}$.

Proposition 10 (operations on $C$-sets). Let $C$ be a character. Let $X, Y$ be two sets.

(i) If $X$ and $Y$ are $C$-sets, then $X \cap Y$ and $X \cup Y$ are $C$-sets.

(ii) If $X$ is a $C$-set and $Y$ is a D-set, then $X \cup Y$ is a $C \cup D$-set and $X \cap Y$ is $a\{C, D\}$-set.

Proposition 11 (fragmentation of $C$-sets). Let $C$ be a $p$ character. Let $X, X_{1}$, and $X_{2}$ be three sets such that $X=X_{1} \cup X_{2}$ and $X_{1} \cap X_{2}=\emptyset$. If $X$ is a $C$-set, then for $1 \leq i \leq 2$, the following conditions are satisfied: (a) $X_{i}$ is a C-set; (b) $M_{X_{i}}^{C} \leq M_{X}^{C}$; and (c) $M_{X_{1}}^{C}+M_{X_{2}}^{C}=M_{X}^{C}$.

Proposition 12 (generalized fragmentation of $P$-sets). Let $P$ be a portrait. Let $X$ be a $P$-set and let $F=\left\{X_{i}\right\}_{1 \leq i \leq m}$ be a partition of $X$. For $1 \leq i \leq m$, the following conditions are satisfied: (a) $X_{i}$ is a P-set; (b) $M_{X_{i}}^{P} \leq M_{X}^{P}$; and $\sum_{i=1}^{i=m} M_{X_{i}}^{P}=$ $M_{X}^{P}(c)$.

3.2. Multisorted Tree-Algebra. This section has two subsections. In Section 3.2.1 we describe the concepts of multisorted tree-algebra as introduced in [21]. Here we start by reminding ourselves of preliminary concepts of multisorted algebra; then we introduce the concept of aggregation operators followed by the concept of valued rooted tree. A multisorted tree-algebra is therefore defined with the intuition of a tree (representing an organisation hierarchy) with multisorted algebra at each node (to represent agents) and aggregation operators at each verse (to represent the flow of information to the top manager). In Section 3.2.2, a matrix-based multisorted tree-algebra is presented as an example of the modeling framework defined in this paper. 
3.2.1. Basic Concepts. The concept of the signature of a many sorted algebra is introduced as well as the concept of many sorted algebra itself. Some auxiliary related notions are defined. The correspondence between (1 sorted) universal algebras [22] and many sorted algebras with one sort only is described by introducing two functions mapping one into the other.

Definition 13 (multisorted signature). A multisorted signature $\Sigma$ is a triplet $\langle S, O, a\rangle$, where

(i) $S$ is a set of sorts;

(ii) $O$ is a set of operation symbols;

(iii) $a: O \rightarrow S^{*} \times S$ is a function, where $S^{*}$ is the set of words from the alphabet $S$ including the empty word.

A multisorted signature is an $S$-set $\Sigma=\left\{\Sigma_{m, s}\right\}_{(m, s) \in S^{*} \times S}$, where $\Sigma_{m, s}$ is the subset of elements $f \in O$ such that $a(f)=$ $(m, s)$. The set $\Sigma_{[], s}$ is called the set of constant symbols of sort $s$.

Definition 14 ( $\Sigma$-multisorted algebra). Let $\Sigma=\langle S, O, a\rangle$ be a multisorted signature. A $\Sigma$-multisorted algebra (or a model for $\Sigma) \mathscr{A}$ consists of the following:

(i) for each $s \in S$, there is a set $A^{s}$ called the carrier;

(ii) for each $f \in O$ such that $a(f)=\left(s_{1} s_{2} \ldots s_{n}, s\right)$, a function $f^{\mathscr{A}}: A^{s_{1}} \times \cdots \times A^{s_{n}} \rightarrow A^{s}$.

\subsubsection{Aggregation Operators. Let $E$ be a nonempty set.}

Definition 15 ( $\Theta$-aggregation operator). Let $\langle E, *\rangle$ be a commutative semigroup. Let $n$ be an integer and $E^{n}$ denote the cartesian product of $E$ defined by $n$-uplets of elements in $E$. Let $E^{\infty}=\bigcup_{n=1}^{\infty} E^{n}$. An aggregation operator on $\langle E, *\rangle$ is a function $\Theta: E^{\infty} \rightarrow E$, called $\Theta$-aggregator or aggregator for short, that assigns to each element $a$ in $E^{\infty}$, an element $\Theta(a)$ of $E$ called the aggregation of $a$ in $\langle E, *\rangle$. For a finite $n$-uplet $X=\left\{x_{1}, x_{2}, \ldots, x_{n}\right\}$ in $E^{n}$ we denote the aggregation $\Theta(X)$ of $X$ by $\Theta_{i=1}^{i=n} x_{i}$.

For example, the symbol $\Sigma$ generally denotes the aggregation operator for the addition operator of finite or infinite elements. The union operator $\bigcup$ is an aggregation operator that constructs the union of a family of sets.

Definition $16((\Sigma, *)$-multisorted algebra). Let $\Sigma=\langle S, O, a\rangle$ be a multisorted signature. Let $*$ be a binary operator. A $(\Sigma, *)$-multisorted algebra (or a model for $(\Sigma, *)) \mathscr{A}$ consists of the following:

(i) for each $s \in S$, there is a commutative semigroup $\left(A^{s}, *\right)$ called the carrier;

(ii) for each $f \in O$ such that $a(f)=\left(s_{1} s_{2} \ldots s_{n}, s\right)$, an homomorphism of commutative semigroup is defined by $f^{\mathscr{A}}: A^{s_{1}} \times \cdots \times A^{s_{n}} \rightarrow A^{s}$.

Definition $17(\Theta$-aggregation of a family of $(\Sigma, *)$-algebra). Let $\langle A, *\rangle$ be a commutative semigroup and let $\Theta$ be an aggregator on $\langle A, *\rangle$. Let $\operatorname{Sub}(\langle A, *\rangle)$ be the set of subcommutative semigroups $\langle X, *\rangle$ of $\langle A, *\rangle$. Let $\left\{A_{i}\right\}_{i \in I}$ be a family of $(\Sigma, *)$ algebras such that for each $i \in I,\left\langle A_{i}, *\right\rangle \in \operatorname{Sub}(\langle A, *\rangle)$. A $\Theta$-aggregation of $\left\{A_{i}\right\}_{i \in I}$ is the $(\Sigma, *)$-algebra denoted by $\Theta\left(\left\{A_{i}\right\}_{i \in I}\right)$ consisting of the following:

(i) $\Theta\left(\left\{A_{i}\right\}_{i \in I}\right)=\left\{a \in A, \forall i \in I, \exists a_{i} \in A_{i}, a=\Theta\left(\left\{a_{i}\right\}_{i \in I}\right)\right\}$;

(ii) for every $f \in \Sigma$ of arity $\left(s_{1} \ldots s_{m}, s\right)$ and for $\left(a_{1}, \ldots\right.$, $\left.a_{m}\right) \in \Theta\left(\left\{A_{i}\right\}_{i \in I}\right)^{s_{1} \cdots s_{m}}$ where for each $j \in\{1, \ldots, m\}$, $a_{j}=\Theta\left(\left\{a_{j i}\right\}_{i \in I}\right)$, the following equation is satisfied:

$$
\begin{aligned}
f & \left(\Theta\left(\left\{a_{1 i}\right\}_{i \in I}\right), \ldots, \Theta\left(\left\{a_{m i}\right\}_{i \in I}\right)\right) \\
& =\Theta\left(\left\{f\left(a_{1 i}, \ldots, a_{m i}\right)\right\}_{i \in I}\right) .
\end{aligned}
$$

3.2.3. $(\Theta, \Delta)$-Valued Rooted Tree. The concept of rooted trees presented below is used to describe hierarchical structures of some organizations. The root is the highest authority and the leaves are the executives.

(1) A tree $\Delta=\left(\Delta_{0}, \Delta_{1}\right)$ is a connected undirected graph with no simple circuits. The set $\Delta_{0}$ is the set of nodes or vertices, and the set $\Delta_{1} \subseteq \Delta_{0} \times \Delta_{0}$ is the set of edges or arcs.

(2) A rooted tree denoted by $\langle\Delta, R\rangle$ is a tree $\Delta$ in which a node $R$ has been designated as the root and every edge is directed away from the root.

(3) Let $\Delta$ be a tree; if $A$ is a node in $\Delta_{0}$ other than the root, the parent or the direct hierarchical superior of $A$ is the unique node $B$ in $\Delta_{0}$ such that there is a directed edge from $B$ to $A$. When $B$ is the parent of $A$, then $A$ is called a child or a direct subordinate of $B$.

(4) Let $\Delta$ be a tree and $A \in \Delta_{0}$ a node of $\Delta$. The ancestors or the hierarchical superiors of $A$ are the nodes in the path from the root to $A$, excluding $A$. The descendants or the subordinates of a node $A$ are those nodes that have $A$ as an ancestor.

(5) A node of a tree $\Delta$ is called leaf, or ground node or operating agent or unit if it has no children. Nodes that have children are called intermediate management agent.

(6) Let $A$ be a node in a tree $\Delta$; the subtree $\Delta_{A}$ of $\Delta$ is a rooted tree with $A$ as its root is the subgraph of the tree consisting of $A$ and all its descendants in $\Delta$.

(7) Let $A$ be a node of a rooted tree $\langle\Delta, R\rangle$; the positive integer $l_{A}$ is the level of $A$ if it is representing the number of edges of the unique path from the root $R$ to $A$. The level of the root of $\Delta$ is equal to zero. The height of a rooted tree $\Delta$ is the maximum of the levels of its nodes.

(8) Let $A$ be a node of a tree $\Delta$, let $l$ be an integer such that $l_{A} \leq l$; the set of nodes of $\Delta$ of level $l$ that are children of $A$ is denoted by $\Delta_{A}^{l}$.

(9) The width $m$ of a tree $\Delta$ is the integer corresponding to the number of leaves in $\Delta$. 
Definition $18((h, m)$-rooted tree). (1) Let $m$ and $h$ be two integers; an $(h, m)$-rooted tree $\langle\Delta, R\rangle$ is a rooted tree with $m$ leaves, for which the level of each leaf is $h$. Each integer $l$, with $0 \leq l \leq h$, is called a hierarchical level of $\Delta$.

(2) Let $\Delta$ be an $(h, m)$-rooted tree, let $l$ be an integer, $0 \leq$ $l \leq h$, and let $A$ be a node of $\Delta$. We denote by $\Delta^{l}$ the set of nodes at the hierarchical level $l$. We denote by $\Delta^{h}$ the set of ground nodes of $\Delta$. We denote by $\Delta_{A}^{h}$ the ground nodes of $\Delta$ that are subordinate to $A$.

Proposition 19. Let $\langle\Delta, R\rangle$ be an $(h, m)$-rooted tree, let $q$ be two integers, and let $A$ be a node of $\Delta$ :

(i) For each integer $l$ such that $0 \leq l<h,\left\{\Delta_{A}^{h}\right\}_{A \in \Delta^{l}}$ is a partition of $\Delta^{h}$.

(ii) For both integers $l$ and $q$ such that $0 \leq l<q \leq h$, $\left\{\Delta_{A}^{q}\right\}_{A \in \Delta^{l}}$ is a partition of $\Delta^{q}$.

Definition 20 (valued rooted tree). Let $V$ be a set of values. An $(h, m)$-valued rooted tree is a triplet $\langle\Delta, S, f\rangle$, where $\langle\Delta, R\rangle$ is an $(h, m)$-rooted tree and $f: \Delta_{0} \rightarrow V$ is a valuation of nodes of $\Delta$.

Definition $21(\Theta$-extension, extensible operation on tree). Let $\langle\Delta, R\rangle$ be an $(h, m)$-rooted graph. Let $\langle E, *\rangle$ be a commutative semigroup for which the associated aggregation operator symbol is $\Theta$. Let $f: \Delta^{h} \rightarrow E$ be a function. The function $\tilde{f}: \Delta_{0} \rightarrow E$ is a $\Theta$-extension to $\Delta_{0}$ of the function $f$ defined on $\Delta^{h}$ when the following are satisfied:

(i) for each $A \in \Delta^{h}, \tilde{f}(A)=f(A)$;

(ii) for each $A \in \Delta_{0}$ such that $A \notin \Delta^{h} ; \tilde{f}(A)=$ $\Theta\left(\{f(S)\}_{S \in \Delta_{A}^{h}}\right)$. to $\Delta_{0}$.

The function $f$ is called a $\Theta$-extensible operation from $\Delta^{h}$

Definition $22((\Theta, \Delta)$-valued rooted tree). Let $\langle E, *\rangle$ be a commutative semigroup with $\Theta$ the associated aggregation operator symbol. A $(\Theta, \Delta)$-valued rooted tree is a triplet $\langle\Delta, R, f\rangle$, where $\langle\Delta, R\rangle$ is an $(h, m)$-rooted tree and $f: \Delta^{h} \rightarrow$ $E$ is a $\Theta$-extensible function from $\Delta^{h}$ to $\Delta_{0}$.

\subsection{4. $(\Sigma, \Theta, \Delta)$-Multisorted Tree-Algebra}

Definition $23((\Sigma, \Theta, \Delta)$-tree-algebra). Let $\langle E, *\rangle$ be a commutative semigroup with $\Theta$ as the associated aggregation operator symbol. Let $\operatorname{Sub}(\langle E, *\rangle)$ be the class of commutative subsemigroups of $\langle E, *\rangle$. Let $\Sigma$ be a signature and $A \lg _{E}(\Sigma)$ be the class of $\Sigma$-algebras with carriers in $\operatorname{Sub}(\langle E, *\rangle)$. Let $\langle\Delta, R\rangle$ be a rooted tree. $\mathrm{A}(\Sigma, \Theta, \Delta)$-multisorted tree-algebra is a tuple $\langle\Sigma, \Theta, \Delta, R, f\rangle$ such that $\langle\Delta, R, f\rangle$ is a $\Theta$-valued rooted tree with $f: \Delta_{h} \rightarrow A \operatorname{Ag}_{E}(\Sigma)$ a $\Theta$-extensible function from $\Delta_{h}$ to $\Delta_{0}$ and such that its $\Theta$-extension $\tilde{f}$ consists of the following:

(i) for each $X \in \Delta^{h}, \tilde{f}(X)=f(X)$; (ii) for each $X \in \Delta_{0}$ such that $X \notin \Delta^{h} ; \tilde{f}(X)=$ $\Theta\left(\{f(S)\}_{S \in \Delta_{A}^{h}}\right)$, is the $\sum$-algebra obtained by $\Theta$ aggregation of the $\sum$-algebras from all subordinated leaves of $X$.

\subsection{5. $(\Sigma, \Theta, \Delta)$-Multisorted Tree Terms, Equations}

Definition 24 ( $\sum$-multisorted terms). Let $S$ be a nonempty set of sorts. Let $X=\left\{X^{s}\right\}_{s \in S}$ be an $S$-set of (distinct) objects called variables. Let $\Sigma=\left\{\Sigma_{m, s}\right\}_{(m, s) \in S^{*} \times S}$ be an $S$-multisorted signature. The $S$-set $T_{\Sigma}(X)=\left\{T_{\Sigma}(X)^{s}\right\}_{s \in S}$ of $\Sigma$-terms over $X$ is the smallest $S$-multisorted set such that

(1) $X^{s} \cup \Sigma_{[1, s} \subseteq T_{\Sigma}(X)^{s}$.

(2) If $\left(t_{1}, \ldots, t_{n}\right) \in T_{\Sigma}(X)^{s_{1} \ldots s_{n}}$ and $f \in \Sigma_{s_{1} \ldots s_{n}, s}$, then the string $f\left(t_{1}, \ldots, t_{n}\right) \in T_{\Sigma}(X)^{s}$.

Definition 25 ( $\Sigma$-multisorted equations and satisfaction). Let $T_{\Sigma}(X)$ be an $S$-multisorted set of $\Sigma$-terms. A $\Sigma$-equation of sort $s \in S$ over $X$ is a couple $\langle p, q\rangle$ of terms from $T_{\Sigma}(X)^{s}$. The couple $\langle p, q\rangle$ is denoted by $\left\langle p\left(x_{1}, x_{2}, \ldots, x_{n}\right), q\left(x_{1}, \ldots, x_{n}\right)\right\rangle$ to emphasise that the set of all variables occurring from either $p$ or $q$ is $x_{1}, \ldots, x_{n}$. A $\sum$-multisorted equation of shape $m=$ $s_{1} s_{2} \cdots s_{n} \in S^{*}$ is a family of $\sum$-equations $\left\{\left\langle p_{s_{i}}, q_{s_{i}}\right\rangle\right\}_{1 \leq i \leq n}$. The set of $\sum$-multisorted equations of shape $s_{1} s_{2} \cdots s_{n} \in S^{*}$ over $X$ is denoted by $E_{\Sigma}(X)^{s_{1} \cdots s_{n}}$.

Let $\langle p, q\rangle$ be a $\sum$-equation of sort $s$ over $X$ containing the variables $x_{1}, x_{2}, \ldots, x_{n}$ of sort $s_{1}, s_{2}, \ldots, s_{n}$, respectively. A $\Sigma$ multisorted algebra $\mathscr{A}$ satisfies $\langle p, q\rangle$ (or the equation $\langle p, q\rangle$ is true in $\mathscr{A}$ or holds in $\mathscr{A}$ ), abbreviated by $\mathscr{A} \vDash\langle p, q\rangle$, if for every choice of elements $\left(a_{1}, a_{2}, \ldots, a_{n}\right)$ from $A^{s_{1}} \times A^{s_{2}} \times \cdots \times$ $A^{s_{n}}$ we have $p\left(a_{1}, a_{2}, \ldots, a_{n}\right)=q\left(a_{1}, a_{2}, \ldots, a_{n}\right)$.

Definition $26((\Sigma, \Theta, \Delta)$-multisorted tree signature). Let $\langle\Delta, R\rangle$ be a rooted tree. A $(\Delta, S)$-multisorted tree signature is a $\Delta_{0}$-indexed family of $S$-multisorted signatures $\left\{\Sigma^{Y}\right\}_{Y \in \Delta_{0}}$. When $\Sigma^{Y}=\Sigma$ for all $y \in \Delta_{0}$, the $(\Delta, S)$-multisorted tree signature is called a $(\Sigma, \Theta, \Delta)$-multisorted tree signature and denoted by $\Delta_{\Sigma, \Theta}$. A $(\Sigma, \Theta)$-multisorted tree signature is a $(\Sigma, \Theta, \Delta)$-multisorted tree signature such that the aggregation operator $\Theta$ is compatible with operation symbols in $\Sigma$.

Definition $27((\Sigma, \Theta, \Delta)$-multisorted set of tree terms). Let $S$ be a nonempty set of sorts. Let $X=\left\{X^{s}\right\}_{s \in S}$ be an $S$-set of (distinct) objects called variables. Let $\Sigma=\left\{\Sigma_{m, s}\right\}_{(m, s) \in S^{*} \times S}$ be an $S$-multisorted signature. Let $\Theta$ be an aggregation operator of $\sum$-multisorted algebra. A $(\Sigma, \Theta, \Delta)$-multisorted tree of term $t^{\Delta}$ is a $\Delta_{0}$-indexed family $\left\{t_{Y}\right\}_{Y \in \Delta_{0}}$ of terms defined as follows:

(1) At each leave node $Y$ in $\Delta^{h}, t_{Y} \in T_{\Sigma}(X)$.

(2) At a node $Y$ such that $\Delta_{h}^{Y}=\left\{Y_{i}\right\}_{i \in I}, t_{Y} \in T_{\Sigma, \Theta, \Delta}^{Y}(X)$ of $(\Sigma, \Theta, \Delta)$-multisorted terms over $X$ is the smallest $S$-multisorted set such that

(a) $X^{s} \cup \Sigma_{[], s} \subseteq T_{\Sigma, \Theta, \Delta}^{Y}(X)^{s}$.

(b) If $\left(t_{1}, \ldots, t_{n}\right) \in T_{\Sigma, \Theta, \Delta}^{Y}(X)^{s_{1} \cdots s_{n}}$ and $f \in \Sigma_{s_{1} \cdots s_{n}, s}$, then the string $f\left(t_{1}, \ldots, t_{n}\right) \in T_{\Sigma, \Theta, \Delta}(X)^{s}$. 
(c) If $\left\{t_{i}\right\}_{i \in I}$ is a family of terms such that $t_{i} \in$ $T_{\Sigma, \Theta, \Delta}^{Y_{i}}(X)^{s}$, then $\Theta\left(\left\{t_{i}\right\}_{i \in I}\right) \in T_{\Sigma, \Theta, \Delta}^{Y}(X)^{s}$.

Definition $28((\Sigma, \Theta, \Delta)$-multisorted tree terms $)$. Let $\mathscr{A}=$ $\langle\Sigma, \Theta, \Delta, R, f\rangle$ be a $S$-multisorted tree-algebra. Let $X$ be an $S$-set of variables $\langle\Delta, \Theta\rangle$. A $(\Sigma, \Theta, \Delta)$-multisorted tree term in a $(\Sigma, \Theta, \Delta)$-multisorted algebra is a tuple $\langle\Sigma, \Theta, \Delta, R, X, t\rangle$ such that $\langle\Delta, R, f\rangle$ is a $\Theta$-valued rooted tree with $t: \Delta_{h} \rightarrow$ $T_{\Sigma}(X)$ a $\Theta$-extensible function from $\Delta_{h}$ to $\Delta_{0}$ such that its $\Theta$-extension $\tilde{f}$ assigns $\Theta$-aggregated terms from the terms in the leaves of $\Delta^{h}$.

\subsection{Matrix-Based Multisorted Tree-Algebra}

Definition 29 (matrix and vectors over a set). Let $E$ be a nonempty set and $n, p$ two positive integers. An $n \times p$-matrix $A$ over $E$, also called a matrix of type $(E, n, p)$, is a function

$$
\begin{aligned}
A:\{1, \ldots, n\} \times\{1, \ldots, p\} & \longrightarrow E, \\
(i, j) & \longmapsto a_{i j} ;
\end{aligned}
$$

we write $A=\left(a_{i j}\right)_{1 \leq i \leq n, 1 \leq j \leq p}$ and the elements $a_{i j}$ are called the components of $A$.

(i) When $n=1$, the matrix $A=\left(a_{11}, \ldots, a_{1 p}\right)$, simply denoted by $A=\left(a_{1}, \ldots, a_{p}\right)$, is a $p$-horizontal vector.

(ii) When $p=1$, then $A=\left(\begin{array}{c}a_{11} \\ \vdots \\ a_{n 1}\end{array}\right)$, simply denoted by $A=\left(\begin{array}{c}a_{1} \\ \vdots \\ a_{n}\end{array}\right)$, is an $n$-vertical vector.

(iii) When $n=p=1$, then $A$ is an element $a$ of $E$.

(iv) When the context is clearly defined, the matrix $A$ is denoted by $\left(a_{i j}\right)$ and the set of all $n \times p$-matrices over $E$ is denoted by $\mathscr{M}(E, n, p)$.

Definition 30 (matrix and vectors operators). Let $(E,+, \times)$ be a field and $n, p$ two positive integers. Let $A=\left(a_{i j}\right)$ and $B=\left(b_{i j}\right)$ be two matrices of $\mathscr{M}(E, n, p), U=\left(u_{j}\right)$ an horizontal vector in $\mathscr{M}(E, 1, p)$, and $V=\left(v_{i}\right)$ a vertical vector in $\mathscr{M}(E, n, 1)$. The following operators are defined:

(1) The sum of matrix operator denoted by + is a function defined as follows:

$$
\begin{aligned}
+: \mathscr{M}(E, n, p) \times \mathscr{M}(E, n, p) & \longrightarrow \mathscr{M}(E, n, p), \\
\left(\left(a_{i j}\right),\left(b_{i j}\right)\right) & \longmapsto\left(a_{i j}+b_{i j}\right) .
\end{aligned}
$$

(2) The row sum of matrix operators denoted by $\vec{f}$ is a function defined as follows:

$$
\begin{aligned}
& \overrightarrow{+}: \mathscr{M}(E, n, p) \longrightarrow \mathscr{M}(E, n, 1), \\
&\left(a_{i j}\right) \longmapsto\left(\begin{array}{c}
\sum_{j=1}^{j=p} a_{1 j} \\
\vdots \\
\sum_{j=1}^{j=p} a_{n j}
\end{array}\right) .
\end{aligned}
$$

(3) The column sum of matrix operators denoted by $\downarrow+$ is a function defined as follows:

$$
\begin{aligned}
\downarrow+: \mathscr{M}(E, n, p) & \longrightarrow \mathscr{M}(E, 1, p), \\
\left(a_{i j}\right) & \longmapsto\left(\sum_{i=1}^{i=n} a_{i 1}, \ldots, \sum_{i=1}^{i=n} a_{i p}\right) .
\end{aligned}
$$

(4) The parallel product of two matrix operators denoted by $\overline{\bar{x}}$ is a function known as Hadamard product and defined as follows:

$$
\begin{aligned}
\overline{\bar{x}}: \mathscr{M}(E, n, p) \times \mathscr{M}(E, n, p) & \longrightarrow \mathscr{M}(E, n, p), \\
\left(\left(a_{i j}\right),\left(b_{i j}\right)\right) & \longmapsto\left(a_{i j} \times b_{i j}\right) .
\end{aligned}
$$

(5) The horizontal product of matrix and vector operator denoted by $\vec{x}$ is a function defined as follows:

$$
\begin{aligned}
\overrightarrow{\times}: \mathscr{M}(E, n, p) \times \mathscr{M}(E, n, 1) & \longrightarrow \mathscr{M}(E, n, p), \\
\left(\left(a_{i j}\right),\left(u_{i}\right)\right) & \longmapsto\left(a_{i j} \times u_{i}\right) .
\end{aligned}
$$

(6) The vertical product of matrix and vector operator denoted by $\downarrow \times$ is a function defined as follows:

$$
\begin{aligned}
& \downarrow \times: \mathscr{M}(E, n, p) \times \mathscr{M}(E, 1, p) \\
& \longrightarrow \mathscr{M}(E, n, p), \\
\left(\left(a_{i j}\right),\left(v_{j}\right)\right) & \longmapsto\left(a_{i j} \times v_{j}\right) .
\end{aligned}
$$

(7) The total sum of a matrix or vector operator denoted by $\oplus$ is a function defined as follows:

$$
\begin{aligned}
\oplus: \mathscr{M}(E, n, p) & \longrightarrow \mathscr{M}(E, 1,1), \\
\left(a_{i j}\right) & \longmapsto \sum_{i=1}^{i=n} \sum_{j=1}^{j=p} a_{i j} .
\end{aligned}
$$

(8) The total product of a matrix or vector operator denoted by $\otimes$ is a function defined as follows:

$$
\begin{aligned}
\otimes: \mathscr{M}(E, n, p) & \longrightarrow \mathscr{M}(E, 1,1), \\
\left(a_{i j}\right) & \longmapsto \prod_{i=1}^{i=n} \prod_{j=1}^{j=p} a_{i j} .
\end{aligned}
$$

One may notice that the operator $\oplus$ is the composition of the two operators $\overrightarrow{+}$ and $\downarrow+$ defined above.

In the rest of this paper, the field $E$ is the field $\mathbb{R}$ of real numbers.

Remark 31. The set $\Sigma=\{\oplus ; \otimes ; \overrightarrow{+} ; \downarrow+; \overrightarrow{+} ; \overline{\bar{x}} ;+; \downarrow \times\}$ is a signature of matrix-based multisorted algebra. 


\section{Hierarchical Multiresource Allocation System Model}

4.1. The Semantic Foundation. The semantics of component models used in this section is based on specific characters and portraits of resources and roles. Each character is a set of finite modalities, whose cardinality is used when defining the matrix dimensions associated with the matrix values domain. The quantitative values could be integers or reals. The characters used to describe the organisation and units' roles are the following two sets:

(i) activity which is the set of all activities defined in the environment;

(ii) task which is the set of all tasks used to define any activity in the environment.

Elsewhere, the characters mainly used to describe organization and unit's resources are the following two sets:

(i) type which is the set of all resource types used in the environment;

(ii) feature which is the set of all resource features that could be used in the environment.

A two-dimensional matrix semantics is said to have the portrait, Task $\times$ Activity, if its rows model the tasks modalities and its columns the activities modalities.

4.2. The Environment Model. The hierarchical resource allocation environment generic model

$$
\begin{aligned}
\mathfrak{E} & =\{\text { Service } ; \mathscr{F} \text { igure } ; \mathscr{H} \text { ierarchy; Operating } \\
& \text { - agent; Management - agent }\}
\end{aligned}
$$

describes the service, resource, hierarchy, operating unit, and management unit of an organization.

\subsubsection{The Service Model}

Definition 32 (service structure, size, and configuration). Let $\mathbf{O}$ be an organisation's name. A model of service of $\mathbf{O}$ is a triplet $\operatorname{Service}(\mathbf{O})=\langle s(\mathbf{O}) ; w(\mathbf{O}) ; c(\mathbf{O})\rangle$ whose components are defined as follows:

(i) $s(\mathbf{O}) \in \mathscr{M}(\mathrm{Bool}, n, p)$ is the matrix of portrait Task $\times$ Activity called the organisation's service structure that defines the task allocated to each organisation's activity in its column when it task line value is not equal to zero.

(ii) $w(\mathbf{O}) \in \mathscr{M}(\mathbb{N}, 1, p)$ is a matrix of portrait Activity called the organisation's service weight that defines the organisation's service size, which is the number of iterations of each of its defined activities.

(iii) $c(\mathbf{O}) \in \mathscr{M}(\mathbb{N}, n, p)$ is a matrix of portrait Task $\times$ Activity called the organization's service configuration defined as $c(\mathbf{O})=s(\mathbf{O}) \downarrow \times w(\mathbf{O})$, which gives for each activity and the corresponding tasks the total number of iterations in the organisation.

\subsubsection{The Resource Figure Model}

Definition 33 (organisation's resource figure). Let $\mathbf{O}$ be an organisation. A resource figure of $\mathbf{O}$ is the matrix Figure $(\mathbf{O}) \in \mathscr{M}(\mathbb{N}, n, q)$ of portrait Type $\times$ Feature that defines the quantity of organisation's resources per type and features.

\subsubsection{The Hierarchy Shape Model}

Definition 34 (organisation's hierarchy shape). Let $\mathbf{O}$ be an organisation. A hierarchy shape of $\mathbf{O}$ is a matrix-based $(\Sigma, \Theta, \Delta)$-tree-algebra $\mathscr{H}$ ierarchy $(\mathbf{O})$, where

(i) $\Delta$ is an $(h, m)$-rooted tree with the leaves in $\Delta^{h}$ as operating agent and the other nodes as management agents that are accountable to their subordinate operating agents. The root is at the top of the hierarchy.

(ii) The matrix-based algebraic signature is: $\Sigma=\{\oplus ; \otimes ; \overrightarrow{+}$; $\downarrow+; \overrightarrow{+} ; \overline{\overline{\times}} ;+; \downarrow \times\}$.

(iii) $\Theta$ is the additional aggregation operator.

\subsubsection{The Operating Agent Model}

Definition 35 (operating agent's role structure, weight, configuration, and figure). Let $\mathbf{O}$ be an organization and $A$ an operating agent of $\mathbf{O}$. Let $\Delta$ be an $(h, m)$-rooted graph that represents the hierarchy of $\mathbf{O}$. Let $s, w, c$, and $f$ be four $\Theta$-extensible functions. An operating agent $A$ is an element of $\mathcal{O}$ perating-agent $(\mathbf{O})$ described by the quadruplet $\langle s(A) ; w(A) ; c(A) ; f(A)\rangle$ whose components are defined as follows:

(i) $s(A) \in \mathscr{M}($ Bool, $n, p)$ is a matrix of portrait Task $\times$ Activity called the operating agent's role structure and defined by the $\Theta$-extensible function $s: \Delta^{h} \rightarrow$ $\mathscr{M}$ (Bool, $n, p)$ which assigns to each operating agent $A$ the matrix that defines the task allocated to each operating agent's activity in its column when its task line value is not equal to zero.

(ii) $w(A) \in \mathscr{M}(\mathbb{N}, 1, p)$ is a matrix of portrait Activity called the operating agent's role weight and defined by the $\Theta$-extensible function $w: \Delta^{h} \rightarrow \mathscr{M}(\mathbb{N}, 1, p)$ which assigns to each operating agent $A$ the matrix that defines the operating agent's role size, which is the number of iterations of each of its defined activities.

(iii) $c(A) \in \mathscr{M}(\mathbb{N}, n, p)$ is a matrix of portrait Task $\times$ Activity called the operating agent's role configuration and defined by the $\Theta$-extensible function $c: \Delta^{h} \rightarrow$ $\mathscr{M}(\mathbb{N}, n, p)$ which assigns to each operating agent $A$ the matrix defined as $c(A)=s(A) \downarrow \times w(A)$ which gives for each activity and the corresponding tasks its total number of iterations in the operating agent.

(iv) $f(A) \in \mathscr{M}(\mathbb{N}, n, q)$ is a matrix of portrait Type $\times$ Feature called operating agent's resource figure and defined by the $\Theta$-extensible function $f: \Delta^{h} \rightarrow$ $\mathscr{M}(\mathbb{N}, n, q)$ which assigns to each operating agent $A$, 
the matrix that defines the quantity of the agent's resources per type and features.

Proposition 36 (fragmentation constraints). Let $\Delta$ be an $(h, m)$-rooted tree and let $\mathbf{O}$ be an organisation with the shape $\Delta$. Let the integer $l$ with $1 \leq l \leq h$ be an hierarchical level and $m_{l}$ the number of agents at the level $l$. We have the following equations:

(i) Fragmentation of the organisation's role: $s(\mathbf{O})=$ $\Theta_{A \in \Delta^{l}} s(A)$. Hence, the family $\left\{s(A), A \in \Delta^{l}\right\} \in$ $\mathrm{Fr}_{m_{l}}(s(\mathbf{O}))$.

(ii) Fragmentation of organisation's weight: $w(\mathbf{O})=$ $\Theta_{A \in \Delta^{l}} w(A)$. Hence, the family $\left\{w(A), A \in \Delta^{l}\right\} \in$ $\mathrm{Fr}_{m_{l}}(w(\mathbf{O}))$.

(iii) Fragmentation of organisation's resources: $f(\mathbf{O})=$ $\Theta_{A \in \Delta^{l}} f(A)$. Hence, the family $\left\{f(A), A \in \Delta^{l}\right\} \in$ $\mathrm{Fr}_{m_{l}}(f(\mathbf{O}))$.

\subsubsection{The Management Agent Model}

Definition 37 (management agent's role structure, weight, configuration, and figure). Let $\mathbf{O}$ be an organization and $A$ an operating agent of $\mathbf{O}$. Let $\Delta$ be an $(h, m)$-rooted graph that represents the hierarchy of $\mathbf{O}$. Let $s, w, c$, and $f$ be four $\Theta$-extensible functions. An operating agent $A$ is an element of Management - agent(O) described by a quintuplet $\left\langle\Delta_{A}^{h}, \widetilde{s}(A) ; \widetilde{w}(A) ; \widetilde{c}(A) ; \widetilde{f}(A)\right\rangle$ whose components are defined as follows:

(i) $\Delta_{A}^{h}=\left\{A_{1}, A_{2}, \ldots, A_{m}\right\}$ is the set of operating agents that are subordinate to $A$.

(ii) $\widetilde{s}(A)=\Theta_{i=1}^{i=m} s\left(A_{i}\right)$ is the matrix of portrait Task $\times$ Activity called the management agent's role structure and defined by the $\Theta$-extension function $\widetilde{s}: \Delta_{0} \rightarrow$ $\mathscr{M}$ (Bool, $n, p)$, which assigns to each management agent $A$, the aggregation of tasks allocated to each of its subordinate operating agent's role structure.

(iii) $\widetilde{w}(A)=\Theta_{i=1}^{i=m} w\left(A_{i}\right)$ is the matrix of portrait Activity called the management agent's role weight and defined by the $\Theta$-extension function $\widetilde{w}: \Delta_{0} \rightarrow$ $\mathscr{M}(\mathbb{N}, 1, p)$, which assigns to each management agent $A$, the aggregation of its subordinate operating agent's role size.

(iv) $\widetilde{c}(A)=\Theta_{i=1}^{i=m} c\left(A_{i}\right)$ is the matrix of portrait Task $\times$ Activity called the operating agent's role configuration and defined by the $\Theta$-extension function $\widetilde{s}: \Delta_{0} \rightarrow$ $\mathscr{M}(\mathbb{N}, n, p)$, which assigns to each management agent $A$ the aggregation of its subordinate operating agents's role configurations.

(v) $\tilde{f}(A)=\Theta_{i=1}^{i=m} f\left(A_{i}\right)$ of portrait Type $\times$ Feature called the operating agent's resource figure and defined by the $\Theta$-extension function $\tilde{f}: \Delta_{0} \rightarrow$ $\mathscr{M}(\mathbb{N}, n, q)$, which assigns to each management agent $A$, the aggregation of its subordinate operating agent's resource figure.
4.3. The Policy Model. The policy model $\mathfrak{P}=$ \{Resource - workload;Activity - workload;Cost convention; Allocation - perspective $\}$ describes the resource workload, the activity workload, the cost convention, and the allocation perspective.

Definition 38 (organisation's resource workload convention). Let $A$ be an operating agent of the hierarchical organization $\mathbf{O}$. The organization's operating agent components are modelled by four matrices defined as follows:

(i) The organisation's resource workload convention is the matrix $\operatorname{res}(\mathbf{O}) \in \mathscr{M}(\mathbb{R}, n, q)$ of portrait, Type $\times$ Feature, that defines the workload allocated to each resource type per feature for a given period.

(ii) The organisation's activity workload convention is the matrix $\operatorname{act}(\mathbf{O}) \in \mathscr{M}(\mathbb{R}, n, p)$ of portrait, Task $\times$ Activity, that defines the workload of each task per activity for a given period.

(iii) The organisation's resource costs convention is the matrix $\operatorname{cost}(\mathbf{O}) \in \mathscr{M}(\mathbb{N}, n, q)$ of portrait, Type $\times$ Feature, that defines the cost of each resource type per feature for a given period.

Definition 39 (organisation's resource allocation policy). The organization's resource allocation policy is a choice that defines the character used to formulate the resource allocation decisions. These characters include, for example, (a) the capacity, when the allocation criteria are based on agent's capacity; (b) the potential, when the allocation criteria are based on agent's potential; (c) the disparity, when the allocation criteria are based one disparity reduction; (d) the workload, when the allocation criteria are based on resources or agent workload; and (e) other relevant criteria.

4.4. The Agent Workload Configuration Model. The agent workload configuration model $\mathfrak{W}=$ \{Operating configuration; Management-configuration $\}$ defines workload configuration of a management or an operating agent as the couple of its workload capacity with its workload potential.

Definition 40 (workload configuration of an operating agent). Let $O$ be a hierarchical organisation. Let $A$ be an operating agent of $O$. The workload capacity, potential, and configuration of $A$ are defined as follows:

(i) The workload capacity of an operating agent $A$ is defined by the $\Theta$-extensible function cap : $\Delta^{h} \rightarrow$ $\mathscr{M}(\mathbb{R}, n, p)$, which assigns to each operating agent $A$ the matrix $\operatorname{cap}(A)=\operatorname{act}(\mathbf{O}) \overline{\overline{\times}} s(A) \downarrow \times w(A)$ of portrait, Task $\times$ Activity, that defines the maximum workload from the structure of $A$.

(ii) The workload potential of an operating agent $A$ is defined by the $\Theta$-extensible function pot : $\Delta^{h} \rightarrow$ $\mathscr{M}(\mathbb{N}, n, q)$ which assigns to each operating agent $A$ the matrix $\operatorname{pot}(A)=f(A) \overline{\overline{\times}} \operatorname{res}(\mathbf{O})$ of portrait, Type $\times$ Feature, that defines the maximum workload of all the resources allocated to $A$. 
(iii) The workload configuration of an operating agent $A$ is defined by the $\Theta$-extensible function conf : $\Delta^{h} \rightarrow$ $\mathscr{M}(\mathbb{R}, n, p) \times \mathscr{M}(\mathbb{N}, n, q)$, which assigns to each operating agent $A$ the couple $\operatorname{conf}(A)=(\operatorname{cap}(A), \operatorname{pot}(A))$ of capacity and potential resource allocation.

Definition 41 (workload configuration of a management agent). Let $\mathbf{O}$ be a hierarchical organisation. Let $A$ be a management unit $\mathbf{O}$ with its subordinate operating units in $\Delta_{A}^{h}=\left\{A_{1}, A_{2}, \ldots, A_{m}\right\}$. The workload capacity, potential, and configuration of $A$ are defined as follows:

(i) The workload capacity of a management agent $A$ is defined by the $\Theta$-extension function $\widetilde{\text { cap }}: \Delta_{0} \rightarrow$ $\mathscr{M}(\mathbb{R}, n, p)$, which assigns to each management agent $A$ the matrix $\widetilde{\text { cap }}(A)=\Theta_{i=1}^{i=m} \operatorname{cap}\left(A_{i}\right)$ of portrait, Task $\times$ Activity, that defines the maximum workload from the aggregation of it subordinate operating units.

(ii) The workload potential of a management agent $A$ is defined by the $\Theta$-extension function $\widetilde{\text { pot }}: \Delta_{0} \rightarrow$ $\mathscr{M}(\mathbb{N}, n, q)$, which assigns to each management agent $A$ the matrix $\widetilde{\operatorname{pot}}(A)=\Theta_{i=1}^{i=m} \operatorname{pot}\left(A_{i}\right)$ of portrait, Type $\times$ Feature, that defines the maximum workload from the aggregation of it subordinate workload.

(iii) The workload configuration of a management agent $A$ is defined by the $\Theta$-extension function $\widetilde{\text { pot }}: \Delta_{0} \rightarrow$ $\mathscr{M}(\mathbb{N}, n, q)$, which assigns to each management agent $A$ the matrix $\widetilde{\operatorname{conf}}(A)=\Theta_{i=1}^{i=m} \operatorname{conf}\left(A_{i}\right)$.

Proposition 42 (fragmentation constraints of agent workload). Let $\Delta$ be an $(h, m)$-rooted tree and let $\mathbf{O}$ be an organisation with the shape $\Delta$. Let the integer l with $1 \leq l \leq h$ be an hierarchical level and $m_{l}$ the number of agents at the level l. We have the following equations:

(i) Fragmentation of the organisation's workload capacity: $\operatorname{cap}(\mathbf{O})=\Theta_{A \in \Delta^{l}} \operatorname{cap}(A)$. Hence, the family $\{\operatorname{cap}(A)$, $\left.A \in \Delta^{l}\right\} \in F r_{m_{l}}(\operatorname{cap}(\mathbf{O}))$.

(ii) Fragmentation of organisation's workload potential: $\operatorname{pot}(\mathbf{O})=\Theta_{A \in \Delta^{l}} \operatorname{pot}(A)$. Hence, the family $\{\operatorname{pot}(A)$, $\left.A \in \Delta^{l}\right\} \in F r_{m_{l}}(\operatorname{pot}(\mathbf{O}))$.

4.5. The Resource Allocation Decision Models. The resource allocation decisions $\mathfrak{D}=\{\mathscr{R}$ eallocation, Reduction; Increase define the reallocation decisions when the organisation's resources remain the same but are redistributed amongst the agents, the reduction decision when some organisation's resources have been removed from the global amount, and the increase decision when more resources have been added to the organisation's resources.

Definition 43 (the resource allocation decisions). Let $\mathbf{O}$ be a hierarchical organisation with an $(h, m)-\Delta$ shape. Let $f(\mathbf{O})$ be its resource figure. Let $f: \Delta^{h} \rightarrow \mathscr{M}(\mathbb{N}, n, q)$ be the $\Theta$-extensible function on $\Delta_{0}$ defining the agent's resource figure state. A resource allocation decision is a function $\alpha$ : $\mathscr{M}(\mathbb{N}, n, q) \rightarrow \mathscr{M}(\mathbb{N}, n, q)$ for which the composition $\alpha \circ f$ with $f$ give another $\Theta$-extensible agent's resource figure. (i) The resource allocation decision $\alpha$ is called reallocation decision when the following condition is satisfied: $\Theta_{A \in \Delta^{h}}(\alpha \circ f)(A)=f(\mathbf{O})=\Theta_{A \in \Delta^{h}} f(A)$. Here the global amount of resource in the organisation has not changed. Only their local allocation figure to agents may have changed.

(ii) The resource allocation decision $\alpha$ is called reduction decision when the following condition is satisfied: $\Theta_{A \in \Delta^{h}}(\alpha \circ f)(A) \leq f(\mathbf{O})=\Theta_{A \in \Delta^{h}} f(A)$. In this case the organisation's amount of resources has been reduced after the decision is completed.

(iii) The resource allocation decision $\alpha$ is called increase decision when the following condition is satisfied: $\Theta_{A \in \Delta^{h}}(\alpha \circ f)(A) \geq f(\mathbf{O})=\Theta_{A \in \Delta^{h}} f(A)$. In this case the organisation's amount of resources has been increased after the decision is completed.

4.6. The Analysis Framework Model. The analysis framework

$$
\mathfrak{A}=\{\mathscr{P} \text { erspective; } \mathscr{D i s p a r i t y} ; \mathscr{C o s t} ; \mathscr{P} \text { roperties }\}
$$

defines the analysis perspective, the disparity criteria, the disparity cost semantics, and the properties.

4.6.1. The Analysis Perspectives. In the analysis perspectives used, the resource allocation is generally based on four aspects: the agent capacity, which is the maximum use of resources by the agent; the agent potential, which is the maximum use of the available resources; the agent performance, which is the actual use of the available resources; and the agent external demand, which is the necessary amount of resources to satisfy the external demand.

4.6.2. The Allocations Disparities. A disparity is a quantitative or qualitative discrepancy between two situations of a given analysis perspective or between two different analysis perspectives. The disparity studied in this paper is the one between agent capacity and potential. In practice, the agent capacity is more stable than its resource figure and resource allocation decision mostly changes the agent's resource figure. Therefore, the workload disparity of an organisation's agent $A$ will be denoted by $\operatorname{disp}(f)(A)$ or $\operatorname{disp}(A)$ for short.

Definition 44 (workload configuration disparities). Let $\mathbf{O}$ be a hierarchical organisation. Let $f: \Delta^{h} \rightarrow \mathscr{M}(\mathbb{N}, n, q)$ be the $\Theta$-extensible function that defines the agent's resource figure. Let $A$ be a management agent $\mathbf{O}$ with its subordinate operating agents in $\left\{A_{1}, A_{2}, \ldots, A_{m}\right\}$. The workload capacity, potential, and configuration of $A$ are defined as follows:

(i) The workload disparity of an operating agent $A$ is defined by the $\Theta$-extensible function $\operatorname{disp}(f): \Delta^{h} \rightarrow$ $\mathscr{M}(\mathbb{R}, n, 1)$, which assigns to each operating agent $A$ the matrix $\operatorname{disp}(f)(A)=(\overrightarrow{+} \operatorname{pot}(A))-(\overrightarrow{+} \operatorname{cap}(A))$ of portrait, Task or Type, that defines for each task the disparity value between the task capacity and resource type potential. 
(ii) The workload disparity of management agent $A$ is defined by the $\Theta$-extension function $\widetilde{\operatorname{disp}(f)}: \Delta_{0} \rightarrow$ $\mathscr{M}(\mathbb{R}, n, 1)$, which assigns to each management agent $A$ the matrix $\widetilde{\operatorname{disp}(f)}(A)=\Theta_{i=1}^{i=m} \operatorname{disp}(f)\left(A_{i}\right)$ of portrait, Task or Type, that defines for each task the disparity value between the task capacity and the task potential.

Definition 45 (workload configuration properties). Let $O$ be a hierarchical organisation. Let $A$ be an operating or management agent in $\mathbf{O}$.

(i) An operating agent is operational workload balanced when $\operatorname{disp}(A)=0$.

(ii) A management agent is managerial workload balanced when $\widetilde{\operatorname{disp}}(A)=0$.

(iii) A hierarchical level $l$ of an organisation is hierarchical workload balanced when all agents at that level are workload balanced.

(iv) An organisation is totally workload balanced when all its operating and management agents have a balanced workload.

(v) When $\operatorname{disp}(A) \neq 0$, then it could be written as the sum of three vectors, $\operatorname{disp}(A)=\operatorname{disp}^{+}(A)+\operatorname{disp}^{-}(A)+$ $\operatorname{disp}^{0}(A)$, where $\operatorname{disp}^{+}(A)$ (resp., $\left.\operatorname{disp}^{-}(A)\right)$ contains the positive values (resp., negative values) of $\operatorname{disp}(A)$.

Remark 46 (workload configuration disparities). Positive values in $\operatorname{disp}^{+}(A)$ represent an excess of resource workload for the agent $A$ while negative values in $\operatorname{disp}^{-}(A)$ represent a deficit of resource workload. The zero values represent balanced workload between resource allocated and agent's capacity for the concerned tasks.

Definition 47 (disparity of allocation decisions). Let $\mathbf{O}$ be an organisation and $f$ its current resource allocation figure. Let $\alpha$ be a resource allocation decision. The disparity of an allocation decision $\alpha$ for an agent $A$ is the disparity of its corresponding allocation figure $\alpha \circ f$ for that agent. The disparity of an allocation decision for an agent $A$ is denoted by $\operatorname{disp}(\alpha)_{A}$ and defined as follows:

$$
\operatorname{disp}(\alpha)_{A}:=\operatorname{disp}(\alpha \circ f)(A) .
$$

Proposition 48 (resource allocation balance-based propositions). Let $\mathbf{O}$ be an organisation and $f$ its current resource allocation figure. Let $\Delta^{l}$ be the set of agents at the hierarchical level l.

(i) If some operating agents are not balanced but have a common ancestor management agent which is balanced, then there is a resource allocation decision such that the concerned operating agent will become balanced after its implementation without using resources from out of their common ancestor.

(ii) If a set of operating agents with the same common ancestor are balanced, then the common ancestor and its subordinate management agents are also balanced. (iii) If a management agent is not balanced, then there is at least one of its subordinate operating and management agents that is not balanced.

(iv) If a management agent is balanced, then there is a resource allocation decision for which subordinate operating agents will become balanced after its implementation.

(v) All the organisation's operating and management agents are balanced if only if the operating agents are balanced.

\subsubsection{An Efficient-Based Resource Allocation Setting in a Multisorted Tree-Algebra Framework}

Definition 49 (a cost-based hierarchical resource allocation setting). Let $\mathbf{O}$ be a hierarchical organisation. A cost-based hierarchical resource allocation setting $S$ is a quintuplet:

$\langle\mathfrak{E}, \mathfrak{P}, \mathfrak{W}, \mathfrak{D}, \mathfrak{A}\rangle$, where $\mathfrak{E}$ is a hierarchical resource allocation environment; $\mathfrak{P}$ is a resource management policy model; $\mathfrak{W}$ is a workload configuration model; $\mathfrak{D}$ is a resource allocation decision model; and $\mathfrak{A}$ is an analysis framework.

4.7. The Problems Formulation Models. In this section we describe two main families of problems that may be studied when analysing a hierarchical resource allocation system. We enumerate in Section 4.7.1 some cost-based decision problems that may be considered when analysing a hierarchical resource allocation decision and in Section 4.7.2 we describe some cost-based optimization problems.

\subsubsection{The Decision Problems}

Definition 50 (the decision problems). Let $\mathbf{O}$ be an organisation.

(i) Let $f: \Delta \rightarrow \mathscr{M}(\mathbb{N}, n, q)$ be an allocation state and let $K$ be a positive real. The disparity reduction problem is defined by the following inequations where the allocation decision $\alpha$ is the variable:

$$
\operatorname{disp}(\alpha \circ f) \leq K \text {. }
$$

(ii) Let $f: \Delta \rightarrow \mathscr{M}(\mathbb{N}, n, q)$ be an allocation state. The disparity decision problem is defined by the following inequations where the allocation decision $\alpha$ is the variable:

$$
\operatorname{disp}(\alpha \circ f) \leq \operatorname{disp}(f) .
$$

(iii) Let $f: \Delta \rightarrow \mathscr{M}(\mathbb{N}, n, q)$ be an allocation state. The disparity reduction allocation choice problem is defined by the following inequation where the allocation decision $g$ is the variable:

$$
\operatorname{disp}(g) \leq \operatorname{disp}(f) .
$$

A decision problem is characterized by the quest to know if a given resource distribution property is achievable. One can define other decision problems depending on the selected objective. 


\subsubsection{The Optimization Problems}

Definition 51 (the optimization problems). Let $\mathbf{O}$ be an organisation.

(i) Let $g: \Delta \rightarrow \mathscr{M}(\mathbb{N}, n, q)$ be an allocation state. The minimum disparity-based allocation choice problem is defined as follows:

$\widehat{f}=\min \{f \in[\Delta, \mathscr{M}(\mathbb{N}, n, q)], \operatorname{disp}(f) \leq \operatorname{disp}(g)\}$.

(ii) Let $f: \Delta \rightarrow \mathscr{M}(\mathbb{N}, n, q)$ be an allocation state. The minimum disparity-based improvement decision problem is defined as follows:

$$
\widehat{\alpha}=\min \left\{\alpha \in \mathscr{M}(\mathbb{N}, n, q)^{2}, \operatorname{disp}(\alpha \circ f) \leq \operatorname{disp}(f)\right\} .
$$

The optimization problem is characterized by the quest to know if an optimum value of a given valuation function of distribution alternatives is achievable.

\subsubsection{The Comparison Problems}

Definition 52 (the gap and distance problems). Let $\mathbf{O}$ be an organisation and let $f$ and $g$ be two resource allocation states. Let $\alpha$ be a resource allocation decision. The gap and distance of allocation state are defined as follows:

(i) The relative gap on the agent $A$ between $f$ and $g$ is defined by

$$
\operatorname{gap}(f, g)(A)=f(A)-g(A) .
$$

(ii) The distance or absolute gap between $f$ and $g$ on the agent $A$ is defined by

$$
\operatorname{dist}(f, g)(A)=|f(A)-g(A)| .
$$

The comparison problems are generally based on criteria to differentiate two resource allocation distributions.

\section{Case Study and Discussions}

5.1. A Case Study of Teachers Allocation Disparity in Cameroon's Secondary School System. Software, named STAPMIS (Secondary Teachers Allocation Performance Measurement Information System), implementing the model has been developed to measure teaching workload disparities (deficit or surplus) and their costs for allocation decisions of Cameroon's secondary school teachers who are civil servants. The management structure of this allocation system has 5 levels: national, regional, divisional, subdivisional, and bottom (corresponding to schools). All the teachers that are civil servants are recruited and paid at the national level. They are put at the disposal of the schools through step-by-step allocation decisions taken at the various hierarchical levels. At each level, the teachers received from the hierarchy are put at the disposal of direct subordinate management units until they are transferred to schools.
TABLE 1: Teaching workload disparities per level.

\begin{tabular}{lcc}
\hline & $\begin{array}{c}\text { Workload deficit } \\
\text { (teaching hours/week) }\end{array}$ & $\begin{array}{c}\text { Workload surplus } \\
\text { (teaching hours/week) }\end{array}$ \\
\hline National level & 94293 & 47510 \\
Regional level & 108413 & 61630 \\
$\begin{array}{l}\text { Divisional } \\
\text { level }\end{array}$ & 129255 & 82472 \\
$\begin{array}{l}\text { Subdivisional } \\
\text { level }\end{array}$ & 144954 & 98171 \\
Bottom level & 180536 & 133753 \\
\hline
\end{tabular}

Data collected in 2014 from secondary schools in eight (out of a total of ten) regions of Cameroon have been analysed to compute, at each level, the teaching workload situation (deficit and surplus). The workload deficit of a school or at a given level corresponds to teaching workload of its needed teachers according to its teaching workload capacity, and the workload surplus corresponds to teaching workload above the teaching workload capacity, which is not used because of overallocation of teachers.

The result of the study is summarized in Table 1 and reveals the following: while the information at the national level shows that the teaching workload deficit is 94293 hours per week and the teaching workload surplus is 47510 hours per week, the real information observed at the schools level is that the teaching workload deficit is 180536 hours per week, and the teaching workload surplus is 133753 hours per week. One should notice that the workload deficit at the schools level is almost double that at the national level while the workload surplus at the schools level is triple that at national level.

This case study shows that, in a hierarchical resource allocation system, (aggregated) information available at a management level does not necessary reflect the reality at the bottom level. Resource allocation decisions based solely on that information, as it is the case of the allocation of teachers in Cameroon's secondary schools, are likely to be nonoptimal.

Our study also reveals that the workload disparities in the system increase and almost double from the top level to the bottom one. Clearly, each management level increases the teachers' allocation disparity within the system. The result is a waste of financial resources, approximately 53 million USD per year, which is huge loss for a poor and developing country like Cameroon.

5.2. Discussions. The research reported in this paper has outlined four results with implications in the various scientific domains concerned with modelling hierarchical multiresource allocation problems as observed in scientific researches $[6,14,23]$. In the domain of resource management policy, the research has provided a generic description of features-based hierarchal multiresource allocation frameworks. This gives an extension to the limitations of computational approaches where resources and recipient agents are simply considered as elements of discrete sets [12]. The centralized computational approaches are considered as 
a features-based approach where the set of features is reduced to a singleton. The study only considers the case where each resource is allocated to only one recipient agent. Elsewhere, the hierarchy usually considered in large organisation studies $[9,24,25]$ is supposed to be balanced in this study. The second result is an approximation formula to measure the workload-based multiresource allocation disparities in hierarchical decision-making systems. It has implications in either resource management when disparity reduction is used as an indicator of quality of services or in economics where the concerned is the cost of disparities as in [26-28].

Even though economic and management semantics of this measure has not been investigated in this paper, one can notice that it could be used as effective and efficient indicators of multiresource allocation decisions as described in [29]. The third result with implications in algebra is the introduction of multisorted tree-algebra concepts combined with concepts of portrait set to build a modelling framework, which can appropriately capture both analytical and computational features of hierarchical multiresource allocation decision systems. It would not have been easy with classic multisorted algebras [30] to combine the description of hierarchy with the algebraic computation required at each node of the decision hierarchy. The fourth result with implications to operation research is the algebraic modelling of disparitybased hierarchical multiresource allocation problems. Even though the resolution of these problems was not our objective, their mathematical formulation opens a way for investigating their resolutions. Elsewhere, these results offer an algebraic specification framework for decision support tools to be inserted when specifying hierarchical multiresource allocation information systems (RAIS).

Finally, the simulation of the model gives the results in Figure 1 which presents a case study result of the disparity throughout the hierarchy. At each node, the goal in the left white box is compared with the result in the right violet box and the resulting value of their difference is in the box below with positive values showing extra resources, negative values showing deficits, and zero value showing balance. For each level, the values on the right help observe the discrepancy between the disparities at the bottom level with those at the intermediary and top levels. For example, while the left node of level L3 is showing a balanced allocation, there are disparities at the subordinate nodes in the lower levels. This shows how aggregated information at the top of the hierarchy (showing here a deficit of 15 hours) does not correspond to realities in the operational level (showing here a deficit of 20 hours and an excess of 5 hours). This justifies the necessity to have a performance measurement information system allowing managers to observe the whole disparity in the system without limiting their observation at their own position.

\section{Conclusion}

In this paper, the research consisted in measuring multiresource distribution disparities and modelling their related reduction problems in hierarchical multiresource allocation decision systems. Despite the research on resource allocation, generally oriented towards computational aspect of social welfare, a modeling framework including the analytical aspects has not yet been formally investigated. To address these issues, a generic description of HMRA system has been done to identify transversal features of such problems. Also, an algebraic modelling framework called multisorted tree-algebra has been introduced to provide a formula that measures workload-based resource distribution disparities and to model their related minimization problems. Though this model is based on our previous description of generic features-based hierarchical multiresource allocation systems, we did not attempt solutions to problems and their related computational studies as this was not the focus of this research. However, the framework proposed opens the way to the investigation of new methods of resolution of this class of problems generally observed in the real world. Also, this framework can be used in formal specification of decision support tools for efficient hierarchical multiresource allocation information systems.

\section{Conflict of Interests}

The authors declare that there is no conflict of interests regarding the publication of this paper.

\section{References}

[1] T. van Zandt, "Hierarchical computation of the resource allocation problem," European Economic Review, vol. 39, no. 3-4, pp. 700-708, 1995.

[2] T. van Zandt, Structure and Returns to Scale of Real-Time Hierarchical Resource Allocation, INSEAD, 2003.

[3] S. E. Humphrey, J. R. Hollenbeck, C. J. Meyer, and D. R. Ilgen, "Hierarchical team decision making," Research in Personnel and Human Resources Management, vol. 21, pp. 175-214, 2002.

[4] J. Ferber, O. Gutknecht, and F. Michel, "From agents to organizations: an organizational view of multi-agent systems," in Agent-Oriented Software Engineering IV: 4th InternationalWorkshop, AOSE 2003, Melbourne, Australia, July 15, 2003. Revised Papers, P. Giorgini, J. Müller, and J. Odell, Eds., vol. 2935 of Lecture Notes in Computer Science, pp. 214-230, Springer, Berlin, Germany, 2004.

[5] N. Altay, "Capability-based resource allocation for effective disaster response," IMA Journal of Management Mathematics, vol. 24, no. 2, pp. 253-266, 2013.

[6] N. Wang, B. Wei, H.-W. Zhang, and J.-D. Wang, "Hierarchical structure resource scheduling model based on grid," in Proceedings of the National Conference on Information Technology and Computer Science (CITCS '12), pp. 998-1003, November 2012.

[7] A. Green, B. Ali, A. Naeem, and D. Ross, "Resource allocation and budgetary mechanisms for decentralized health systems: experiences from Balochistan, Pakistan," Bulletin of the World Health Organization, vol. 78, no. 8, pp. 1024-1035, 2000.

[8] M. Bennour, D. Crestani, and O. Crespo, "Une méthode d'affectation des ressources humaines aux processus industriels," Journal Européen des Systèmes Automatisés, vol. 42, no. 5, pp. 541-577, 2008.

[9] M. Zeynalian, G. Jandaghi, A. Memariani, and H. Jahanshahi, "Designing a multi-purpose optimization model for budget allocation using a hierarchical approach," European Journal of 
Economics, Finance and Administrative Sciences, no. 25, pp. 126$135,2010$.

[10] W. Ogryczak, A. Wierzbicki, and M. Milewski, "A multi-criteria approach to fair and efficient bandwidth allocation," Omega, vol. 36, no. 3, pp. 451-463, 2008.

[11] T. Ensor, H. Firdaus, D. Dunlop et al., "Budgeting based on need: a model to determine sub-national allocation of resources for health services in Indonesia," Cost Effectiveness and Resource Allocation, vol. 10, article 11, 2012.

[12] Y. Chavaleyre, P. E. Dunne, U. Endriss et al., "Issues in multiagent resource allocation," Informatica, vol. 30, no. 1, pp. 3-31, 2006.

[13] C. Joe-Wong, S. Sen, T. Lan, and M. Chiang, "Multi-resource allocation: Fairness-efficiency tradeoffs in a unifying framework," in Proceedings of the IEEE Conference on Computer Communications (INFOCOM '12), pp. 1206-1214, Orlando, Fla, USA, March 2012.

[14] S. Zikos and H. D. Karatza, "Resource allocation strategies in a 2-level hierarchical grid system," in Proceedings of the 41st Annual Simulation Symposuim, pp. 157-164, April 2008.

[15] H. Lee, B. Lee, K. Park, and R. Elmasri, "Fusion techniques for reliable information: a survey," International Journal of Digital Content Technology and Its Applications, vol. 4, no. 2, pp. 74-88, 2010.

[16] H. Boström, S. F. Andler, M. Brohede et al., "On the definition of information fusion as a field of research," Tech. Rep. HS-IKITR-07-006, Informatics Research Centre, University of Skövde, 2007.

[17] T. Van Zandt, "Real-time hierarchical resource allocation," Discussion Papers 1231, Northwestern University, Center for Mathematical Studies in Economics and Management Science, 1997, http://www.kellogg.northwestern.edu/research/ math/papers/1231.pdf.

[18] M. Bennour, Contribution à la modélisation et à l'affectation des ressources humaines dans les processus [Thèse de doctorat], Université de Montpellier 2, 2004.

[19] D. Simba, N. Mwangu, and G. Msamanga, "Rationalizing human resource deployment in the wake of reforms: the need for measuring health workers workload," Tanzania Medical Journal, vol. 19, no. 2, 2004.

[20] J. M. Bryson, F. Ackermann, and C. Eden, "Putting the resourcebased view of strategy and distinctive competencies to work in public organizations," Public Administration Review, vol. 67, no. 4, pp. 702-717, 2007.

[21] E. P. Zobo and N. M. Fouda, "Multisorted tree algebra," Applied and Computational Mathematics, vol. 3, no. 6, pp. 295-302, 2014.

[22] K. Denecke and S. L. Wismath, Universal Algebra and Applications in Theoretical Computer Science, Chapman \& Hall, CRC Press, 2002.

[23] M. S. Levin and M. A. Danieli, "Hierarchical decision making framework for evaluation and improvement of composite systems (example for building)," Informatica, vol. 16, no. 2, pp. 213240, 2005.

[24] M. D. Lee, "A hierarchical Bayesian model of human decisionmaking on an optimal stopping problem," Cognitive Science, vol. 30, no. 3, pp. 1-26, 2006.

[25] W.-C. Huang, J.-Y. Teng, and M.-C. Lin, “The budget allocation model of public infrastructure projects," Journal of Marine Science and Technology, vol. 18, no. 5, pp. 697-708, 2010.

[26] W.-H. Tsai, "Quality cost measurement under activity-based costing," International Journal of Quality \& Reliability Management, vol. 15, no. 7, pp. 719-752, 1998.
[27] R. Buyya, D. Abramson, J. Giddy, and H. Stockinger, "Economic models for resource management and scheduling in grid computing," Concurrency Computation: Practice and Experience, vol. 14, no. 13-15, pp. 1507-1542, 2002.

[28] H.-M. Lee, J.-S. Su, and C.-H. Chung, "Resource allocation analysis model based on grid environment," International Journal of Innovative Computing, Information and Control, vol. 7, no. 5, pp. 2099-2108, 2011.

[29] M. Engwall and A. Jerbrant, "The resource allocation syndrome: the prime challenge of multi-project management?" International Journal of Project Management, vol. 21, no. 6, pp. 403-409, 2003.

[30] J. Circulis, "A first-order logic for multi-algebras," in Proceedings of the Novi Sad Algebraic Conference, vol. 34, pp. 27-36, 2004. 


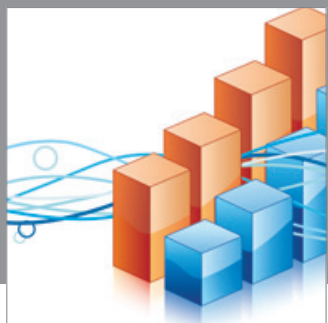

Advances in

Operations Research

mansans

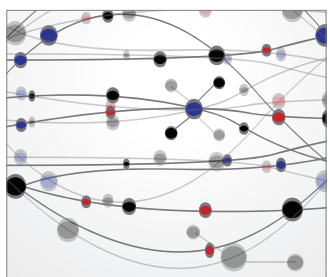

The Scientific World Journal
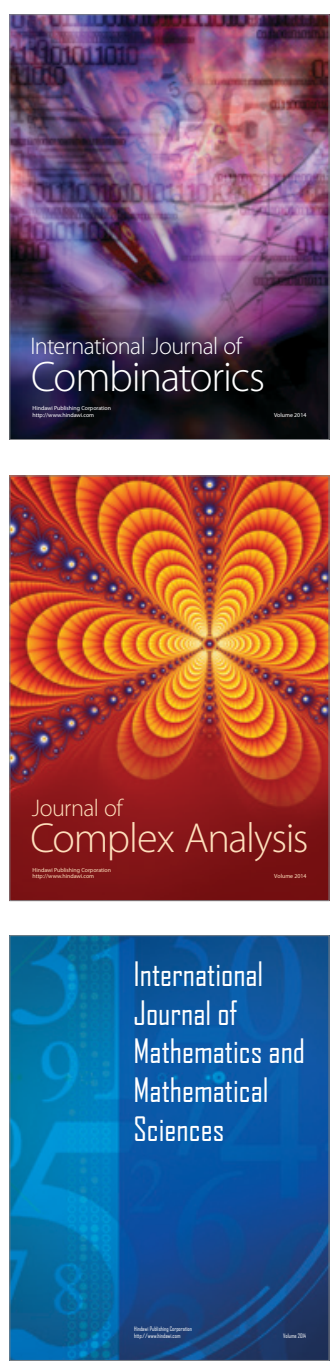
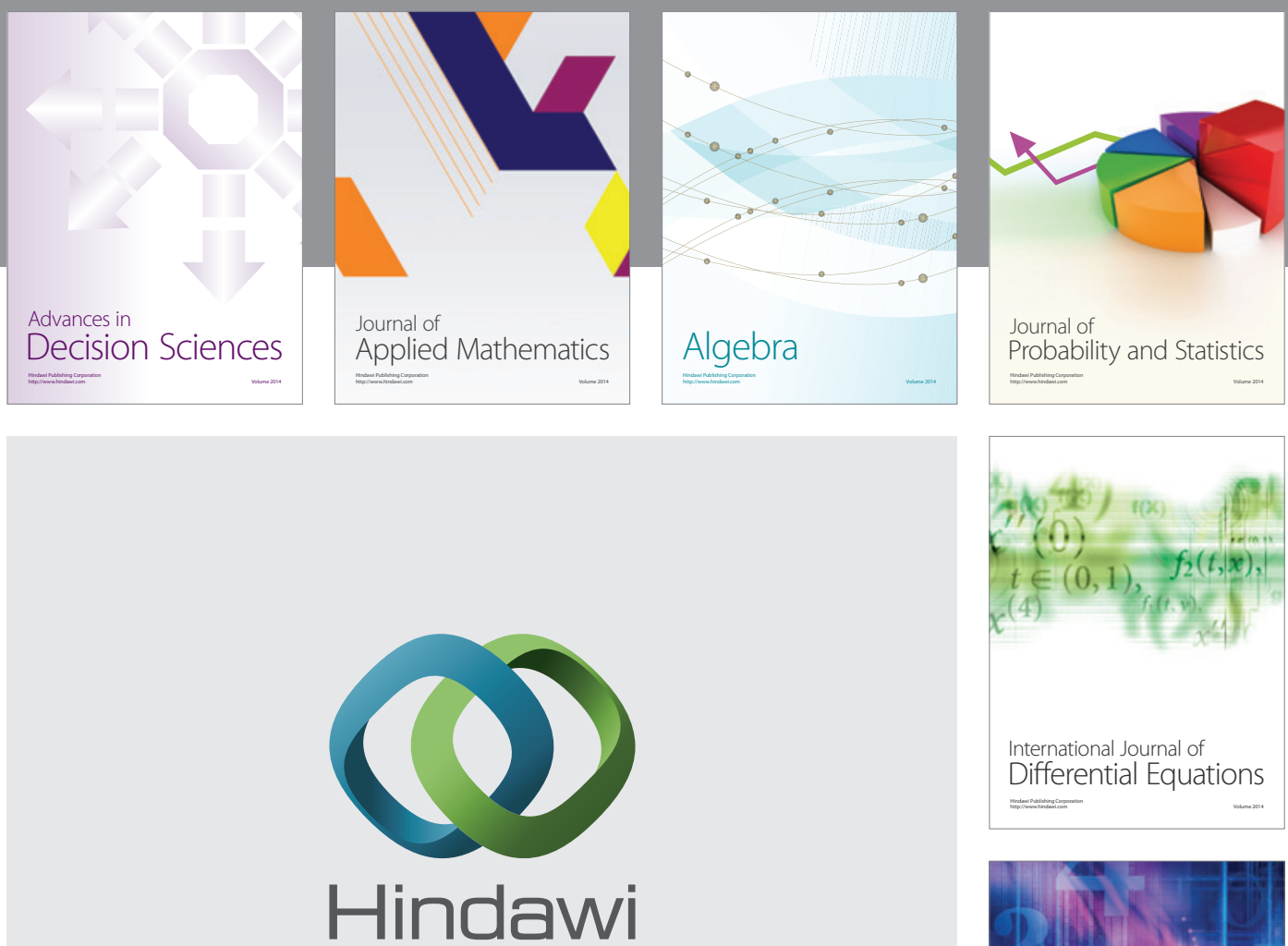

Submit your manuscripts at http://www.hindawi.com
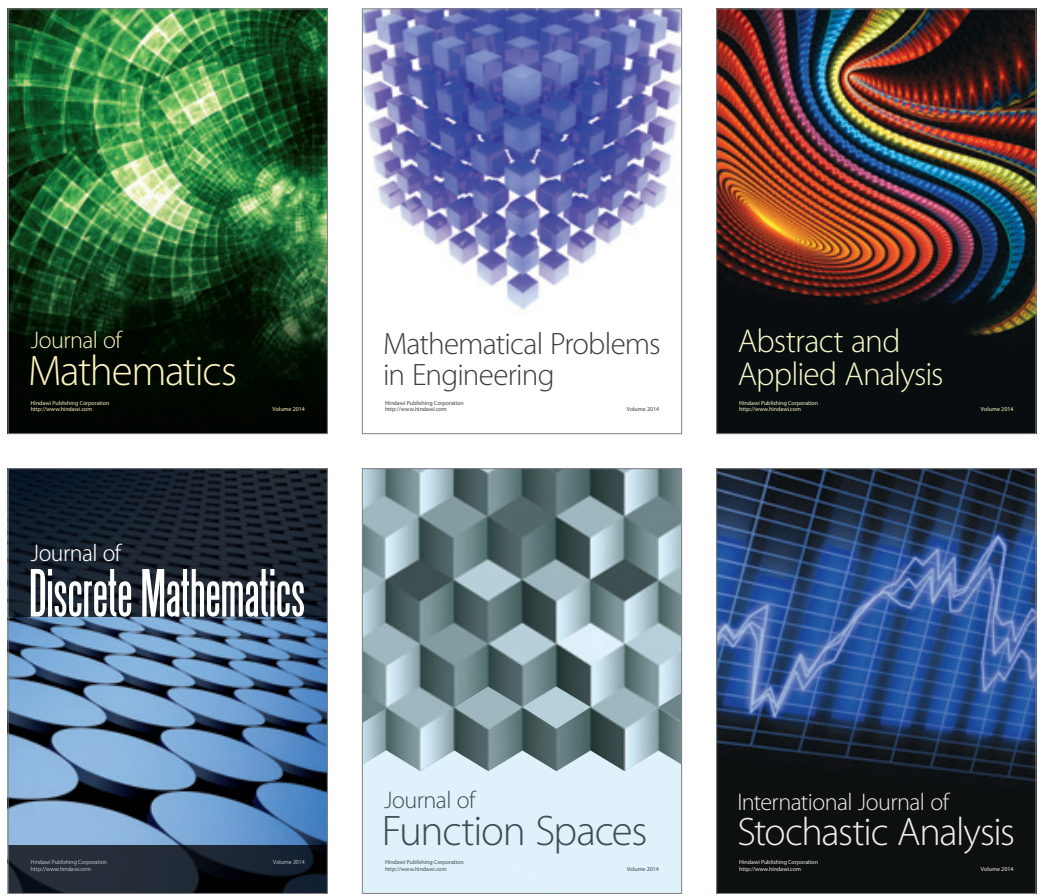

Journal of

Function Spaces

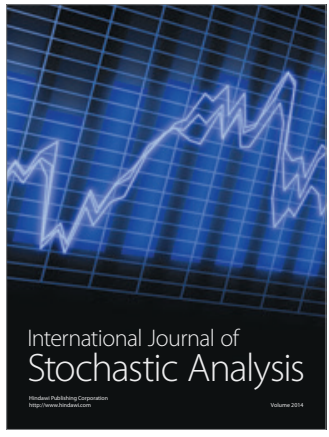

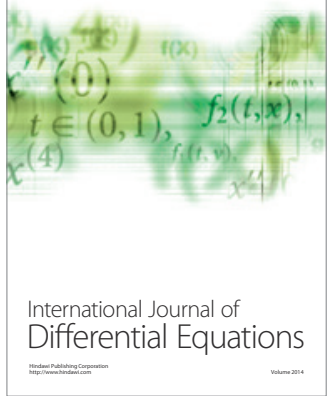
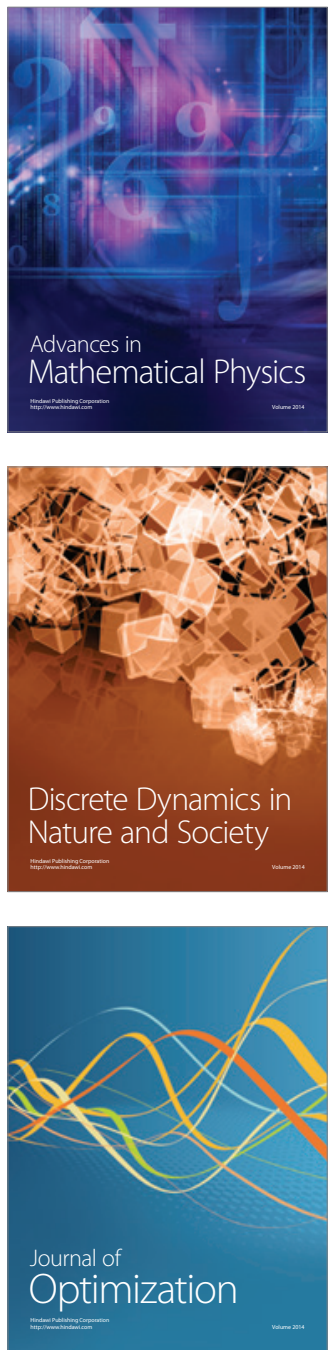